\title{
KNOWLEDGE OF PRE-PREGNANCY CARE AMONG WOMEN WITH HIGH RISK PREGNANCY IN PERAK, MALAYSIA: WHAT ARE THE FACTORS?
}

\author{
Nazirah Jusoh ${ }^{1,2}$, Tengku Alina Tengku Ismail ${ }^{1}$ and Noor Aman A. Hamid ${ }^{1}$ \\ ${ }^{1}$ Department of Community Medicine, School of Medical Sciences, Universiti Sains Malaysia, 16150 Kubang Kerian, \\ Kelantan, Malaysia. \\ ${ }^{2}$ Pahang State Health Office, Jalan IM 4, Bandar Indera Mahkota, 25582 Kuantan, Pahang, Malaysia.
}

Corresponding author: Tengku Alina Tengku Ismail

Email: dralina@usm.my

\begin{abstract}
Pre-pregnancy care is an important approach for maternal and child health. This study aimed to determine the knowledge about pre-pregnancy care and factors associated with good pre-pregnancy care knowledge among women with high risk pregnancy in Kinta, Perak. A cross-sectional study was conducted among women diagnosed as high-risk pregnancy in governmental health clinics in Kinta. Stratified random sampling was applied and interviewer-guided questionnaires were administered. A proforma and the validated Pre-pregnancy Care Knowledge and Practice Questionnaire were used, consisting of 21 items on knowledge. Good pre-pregnancy care knowledge was defined as those with the total score of more than 12. Logistic regression analysis was conducted using SPSS software. Of the 490 women, their mean age was 29.98 (SD 5.51) years. Less than half (48.6\%) had good knowledge of pre-pregnancy care. Education level and attended pre-pregnancy care services were significantly associated with good pre-pregnancy care knowledge. Compared to women with secondary and below education, those with tertiary education had two times odds of having good pre-pregnancy care knowledge (Adjusted odds ratio 2.06; 95\% Cl: 1.41, 3.03; p<0.001). Compared to women who never attended pre-pregnancy care services, those who had attended were almost 2.5 times higher odds of having good pre-pregnancy care knowledge (Adjusted odds ratio 2.46; 95\% Cl: 1.59, 3.83; p<0.001). The prepregnancy care knowledge of women with high risk pregnancy in this study was still unsatisfactory. The area with a lacking of knowledge and the significant factors should be focused to improve pre-pregnancy care knowledge and utilization.
\end{abstract}

Keywords: Pre-pregnancy care; knowledge; high risk pregnancy; pre-pregnancy care services.

\section{INTRODUCTION}

Every woman should receive quality antenatal care throughout pregnancy, which includes health promotion, screening and diagnosis, and disease prevention in order to save and improve lives, healthcare utilization and quality of care $^{1}$. However, women who started antenatal care late may lead to delay in detecting and managing possible risk factors and medical problems. Thus, improving maternal health before conception through pre-pregnancy care is increasingly recognised as an important public health strategy. Pre-pregnancy care is defined by the World Health Organization as "...the provision of biomedical, behavioural and social health interventions to women and couples before conception occurs. It aims at improving their health status and reducing behavioural and individual and environmental factors that contribute to poor maternal and child health outcomes..."2. Prepregnancy care was associated with reduced risk of preterm birth, low birth weight, as well as maternal and neonatal complications ${ }^{3}$. Thus, prepregnancy care should be targeted to all women, in addition to the routine antenatal care that they received after conception.

All women in reproductive age group should receive pre-pregnancy care. Women with medical problems or risk factors should receive prepregnancy care to plan their pregnancy and optimize their health conditions at least three months prior to conception ${ }^{4}$. Among the prepregnancy risk factors include 1) women aged less than 18 or above 35 years old, 2) having negative lifestyle conditions such as smoking, high risk sexual behaviour and overweight/obesity, and 3) specific risk factors of obstetric history, medical history, medications, surgical history, family history, social history, and vaccination ${ }^{5}$. A study conducted in Selangor, Malaysia found that $68.8 \%$ of women screened in pre-pregnancy clinics had at least one risk factor; with $35.0 \%$ were overweight and obese, and $14.0 \%$ had anaemia ${ }^{6}$. If these risk factors are not adequately intervened prior to conception, the women will enter pregnancy state with various health problems. Among women who attended primary health clinics for antenatal follow-up in Selangor, 28.0\% of them were classified as high risk pregnancy, following the risk assessment system of Malaysian Ministry of Health ${ }^{5,7}$. Therefore, pre-pregnancy care should be emphasized to reach all women in the reproductive age group.

In Malaysia, pre-pregnancy care was formalized into the health care services since $2002^{5}$. In general, the target groups were 1) prospective couples intending to get married, 2) married 
women who planned for a pregnancy, and 3) women in the reproductive age group. Those with medical problems and risk factors were included as the specific target groups. Screening, identification and appropriate management and referral of the risk factors and medical problems were the main activities under this service. Since pre-pregnancy care has been introduced for almost ten years in all government health clinics in Malaysia, it is important to identify the women's knowledge related to it. Furthermore, knowledge has been shown to be an important factor influencing women to utilize the service ${ }^{8-9}$.

Even though women involved in a qualitative study in UK knew about pre-pregnancy activities such as quitting smoking and consuming healthy diet, the information was not at the forefront of their minds and only expressed upon prompting ${ }^{10}$. In Ethiopia, 35.4\% of reproductive-aged women have heard about pre-pregnancy care, with only $17.3 \%$ had good knowledge 9 . Among women with chronic health conditions, their knowledge on pre-pregnancy care benefits, information sources and availability of the service were found to have significant gaps ${ }^{11}$. The main source of information was the health care providers 9,12 . However, another study in Ethiopia found almost half of the health care providers had poor knowledge about pre-pregnancy care $^{13}$.

In Malaysia, a qualitative study conducted among married women in reproductive age group found that majority of the respondents had heard about pre-pregnancy care, but a handful of them did not know the existence of this services in government clinics or hospitals ${ }^{14}$. Interviews conducted with health care providers also highlighted their concerns that women were unaware or having lack of knowledge on pre-pregnancy issues that subsequently acted as a barrier to pre-pregnancy care $^{15}$. Thus, it is important to explore the level of knowledge among women regarding prepregnancy care, and specifically identify the areas that were lacking, to facilitate further improvement of the current health promotion strategies. This is of value considering the limited information on pre-pregnancy care knowledge among women in Malaysia. Furthermore, focusing on women with high risk pregnancy was crucial, acknowledging the burden of chronic diseases and other risk factors among women in Malaysia. It is hoped that pre-pregnancy care practice will also be improved and subsequently lead to better maternal and child health outcomes. This study aimed to determine the knowledge of women with high risk pregnancy about pre-pregnancy care and the factors associated with good pre-pregnancy care knowledge.

\section{METHODS}

\section{Study design and population}

This is a cross-sectional study conducted from March 2016 to March 2017 in Kinta district, Perak. Kinta has multiracial population with almost equal proportion of Malay, Chinese and Indian ethnicities. The study was conducted in all 14 governmental health clinics with maternal and child health services in this district.

The study was registered with National Medical Research Register (ID number: NMRR15-195928396). Ethical approval was obtained from the Human Research Ethics Committee Universiti Sains Malaysia (USM/JEPeM/15090296) and Medical Research \& Ethics Committee, Ministry of Health Malaysia [(7)KKM/NIHSEC/P15-1580], which complies with the Declaration of Helsinki.

The study population was women diagnosed as high risk pregnancy during their first antenatal check-up (booking visit) in governmental health clinics in Kinta District during the study period. The criteria of high risk pregnancy are based on the presence of any of the following ${ }^{5}$ : i.Current or previous history of hypertension, diabetes, cardiovascular disease, congenital heart disease, thyroid disease, connective tissue disease, systemic lupus erythematosus, chronic kidney disease, preeclampsia or gestational diabetes.ii.Overweight and obesity.iii.Severe anemia in previous or current pregnancy (haemoglobin level is less than $7 \mathrm{~g} / \mathrm{dl}$ ). iv. Age $\leq 19$ years or more than 35 years, which is more likely to be associated with morbidities and mortality.

The exclusion criteria were women who were not able to communicate in either the Malay or English language, or those with known mental health problems.

\section{Sample size and sampling method}

Sample size was calculated to answer all the objectives. The biggest sample size was derived from single proportion formula. Taking a precision of 0.05 at a $95 \%$ confidence interval, the required sample size determined by a single proportion formula based on $51.9 \%$ women with good prepregnancy care knowledge was 495 (including 30\% possibility of non-response rate) ${ }^{16}$

A stratified random sampling with number of samples proportionate to size was used. There are 14 health clinics with maternal and child health services, and the estimated number of high risk pregnancy cases in each clinic ranged from 40 to 100 cases per year. The proportion of selected respondents was based on the percentages of high risk pregnancy obtained from the registration. They ranged from a minimum of 30 respondents 
to a maximum of 45 respondents randomly selected from every health clinic.

\section{Research tool and data collection}

A proforma and the Pre-pregnancy Care Knowledge and Practice Questionnaire were used $^{17}$. The Malay language questionnaire was developed in another state in Malaysia with the same population ethnic background. The Cronbach's alpha of the validated questionnaire was 0.79. Permission was obtained from the author prior to its use.

There were 21 items in knowledge domain, assessing the following: a) examples of conditions classified as high risk pregnancy (4 items), b) risk of poor birth spacing (4 items), c) recommended duration of birth spacing (3 items), d) diet during pregnancy (4 items), e) effect of smoking (1 item), f) folic acid supplementation (1 item), g) risk of maternal anaemia (4 items). They used a three-point Likert scale (yes/no/not sure) response options, with a score of " 1 " for correct answers, and incorrect and not sure responses were scored " 0 ". The total score of more than 12 was categorized as good pre-pregnancy care knowledge and 12 or less was considered as poor knowledge ${ }^{17}$.

In addition, a proforma which consists of sociodemographic data, obstetric information and prepregnancy care information was also used, to obtain data on the factors associated with good pre-pregnancy care knowledge. The prepregnancy care information assessed whether they have ever received advice on pre-pregnancy care, referred for pre-pregnancy care services, and attended the services at least once prior to the current pregnancy.

During data collection, the selected women were informed about the study and their written consent were obtained. The interviewer-guided questionnaires were administered at a convenience place in the health clinics. In average, the questionnaire took about 15 minutes to be answered by the respondents.

\section{Statistical analysis}

Data was entered and analysed using IBM SPSS statistics version 22 software. All data were tabulated for descriptive statistics. The categorical variables were summarized in frequency (n) and percentage (\%). The numerical variables were described in mean and standard deviation (SD) or median and interquartile range (IQR) depending on normality of distribution. The percentage of women with good pre-pregnancy care knowledge was calculated.
Logistic regression analysis was used to determine factors associated with good pre-pregnancy care knowledge. The dependent variable was good knowledge, and the independent variables were socio-demographic characteristics, obstetric data, and pre-pregnancy care information. The associations between each of these variables with the dependent variable were assessed using simple logistic regression analysis. All variables with $\mathrm{p}<0.25$ from this univariable analysis, or clinically significant variables were selected based on Forward LR and Backward LR methods into multiple logistic regression analysis. The final model was presented with adjusted odds ratio and 95\% confidence interval $(\mathrm{Cl})$, Wald statistics and $\mathrm{p}$-value. The level of significance was set at pvalue of less than 0.05 .

\section{RESULTS}

Table 1 shows the socio-demographic characteristics of 490 respondents in this study. Their mean age was 29.98 (SD 5.51) years, with the youngest being 16 years old and the eldest was 43 years old. The majority of the respondents were from Malay ethnicity, 347 (70.8\%). Ninetyeight percent of them were married, and more than half $(55.7 \%)$ were employed. The median distance from their resident places to the health clinic was 3.50 (IQR 2.50) km.

Table 2 presents obstetric characteristics of the respondents. Seventy-four percent of them had their first antenatal visit at or before 12 gestational weeks. A majority of them, 331 $(67.6 \%)$ were parity 1 to 4 , while 151 (30.8\%) were nulliparous women. Fifty-five percent of the respondents had previous history of pregnancy complications, of which around 35\% experienced miscarriage, gestational diabetes mellitus and lower segment caesarean section, respectively. Almost $40.0 \%$ of them had an unplanned pregnancy, with only $24.9 \%$ practised family planning prior to this pregnancy.

The mean (SD) score for knowledge of prepregnancy care was 12.21 (3.92). Less than half of the respondents had good knowledge of prepregnancy care, 238 (48.6\%). Table 3 presents the percentages of correct responses for each item assessing knowledge of pre-pregnancy care. Around $80.0 \%$ of the respondents answered correctly on items assessing diet during pregnancy. Majority of them (93.0\%) knew that smoking can cause harm to the baby. However, around $30.0 \%$ of the respondents wrongly answered that birth spacing of one year is recommended, as well as almost $60.0 \%$ of them stated that is it recommended to space birth for more than five years. In addition, more than half of them did not know the conditions considered as high risk pregnancy, especially maternal 
underweight $(70.4 \%)$, teenage pregnancy $(48.4 \%)$ and advanced maternal age (44.1\%).

Simple logistic regression analysis showed that education level, employment status, monthly household income, family planning practice, received advice on pre-pregnancy care, referred for pre-pregnancy care services, and attended pre-pregnancy care services were significantly associated with good pre-pregnancy care knowledge (Table 4). All variables with $p<0.25$ from this univariable analysis were selected for multiple logistic regression analysis using Forward LR and Backward LR methods. These variables include education level, employment status, monthly household income, parity, previous history of pregnancy complications, family planning practice, received advice on pre- pregnancy care, referred for pre-pregnancy care services, and attended pre-pregnancy care services.

After controlling for other variables, education level and attended pre-pregnancy care services were significantly associated with good prepregnancy care knowledge (Table 5). Compared to women with secondary and below education, those with tertiary education had two times odds of having good pre-pregnancy care knowledge (Adjusted OR 2.06; 95\% Cl: 1.41, 3.03; $p<0.001$ ). Compared to women who never attended prepregnancy care services, those who had attended were almost 2.5 times higher odds of having good pre-pregnancy care knowledge (Adjusted OR 2.46; $95 \% \mathrm{Cl}: 1.59,3.83 ; p<0.001)$

Table 1: Socio-demographic characteristics of the respondents $(n=490)$

\begin{tabular}{|c|c|}
\hline Variables & $\mathrm{n}(\%)$ \\
\hline Age (year) & $29.98(5.51)^{\mathrm{a}}$ \\
\hline Less than 20 & $18(3.7)$ \\
\hline 20 to 35 & $396(80.8)$ \\
\hline More than 35 & $76(15.5)$ \\
\hline \multicolumn{2}{|l|}{ Ethnicity } \\
\hline Malay & $347(70.8)$ \\
\hline Chinese & $65(13.3)$ \\
\hline Indian & $74(15.1)$ \\
\hline Others & $4(0.8)$ \\
\hline \multicolumn{2}{|l|}{ Marital status } \\
\hline Unmarried & $9(1.8)$ \\
\hline Married & $481(98.2)$ \\
\hline \multicolumn{2}{|l|}{ Education level } \\
\hline Secondary and below & $313(63.9)$ \\
\hline Tertiary & $177(36.1)$ \\
\hline \multicolumn{2}{|l|}{ Employment status } \\
\hline Unemployed & $217(44.3)$ \\
\hline Employed & $273(55.7)$ \\
\hline Monthly household income (RM) & $3000.00(3000.00)^{b}$ \\
\hline Less than 5000 & $353(72.3)$ \\
\hline 5000 to 10,000 & $119(24.4)$ \\
\hline More than 10,000 & $16(3.3)$ \\
\hline Distance from health clinic $(\mathbf{k m})$ & $3.50(2.50)^{\mathrm{b}}$ \\
\hline \multicolumn{2}{|c|}{ Transportation problems to health clinic } \\
\hline Yes & $59(12.0)$ \\
\hline No & $431(88.0)$ \\
\hline
\end{tabular}

$\overline{a^{a} \text { mean }(S D),{ }^{b} \text { median (IQR) }}$ 
Table 2: Obstetric characteristics of the respondents $(n=490)$

\begin{tabular}{|c|c|}
\hline Variables & $\mathrm{n}(\%)$ \\
\hline Gestational age at booking (weeks) & $10.00(5)^{a}$ \\
\hline 12 weeks and below & $364(74.3)$ \\
\hline More than 12 weeks & $126(25.7)$ \\
\hline Parity & $1.00(2.00)^{b}$ \\
\hline Nulliparous & $151(30.8)$ \\
\hline Parity 1 to 4 & $331(67.6)$ \\
\hline Parity 5 and above & $8(1.6)$ \\
\hline \multicolumn{2}{|l|}{ Previous history of pregnancy complications } \\
\hline No & $217(44.3)$ \\
\hline Yes & $273(55.7)$ \\
\hline \multicolumn{2}{|l|}{ Types of pregnancy complications* $(n=273)$} \\
\hline Miscarriage & $98(35.9)$ \\
\hline Induced abortion & $1(0.4)$ \\
\hline Ectopic pregnancy & $7(2.6)$ \\
\hline Gestational diabetes mellitus & $96(35.1)$ \\
\hline Pregnancy-induced hypertension & $46(16.8)$ \\
\hline Congenital heart disease & $2(0.7)$ \\
\hline Cardiovascular disease & $1(0.4)$ \\
\hline Thyroid disease & $8(2.9)$ \\
\hline Renal disease & $3(1.1)$ \\
\hline Postpartum haemorrhage & $1(0.4)$ \\
\hline Perinatal death & $9(3.3)$ \\
\hline Congenital malformation & $3(1.1)$ \\
\hline Instrumental delivery & $30(11.0)$ \\
\hline Caesarian section & $82(30.0)$ \\
\hline \multicolumn{2}{|l|}{ Planned pregnancy } \\
\hline No & $192(39.2)$ \\
\hline Yes & $298(60.8)$ \\
\hline \multicolumn{2}{|l|}{ Family planning practice prior to current pregnancy } \\
\hline No & $368(75.1)$ \\
\hline Yes & $122(24.9)$ \\
\hline Items & n (\%) \\
\hline \multicolumn{2}{|l|}{ 1. Risk for high risk pregnancy } \\
\hline a) Age below 18 years & $253(51.6)$ \\
\hline b) Small body size & $145(29.6)$ \\
\hline c) First pregnancy at age 35 years and above & $274(55.9)$ \\
\hline d) Twin pregnancy & $325(66.3)$ \\
\hline \multicolumn{2}{|l|}{ 2) Risk of poor birth spacing } \\
\hline a) Maternal anaemia & $353(72.0)$ \\
\hline b) Congenital malformation & $126(25.7)$ \\
\hline c) Premature labour & $196(40.0)$ \\
\hline d) Postpartum haemorrhage & $235(48.0)$ \\
\hline \multicolumn{2}{|l|}{ 3) Recommendation for good birth spacing practices } \\
\hline a) One year & $352(71.8)$ \\
\hline b) Between 2 to 4 years & $439(89.6)$ \\
\hline c) More than 5 years & $215(43.9)$ \\
\hline \multicolumn{2}{|l|}{ 4) Knowledge of diet during pregnancy } \\
\hline a) eat a balanced diet but more frequently than non-pregnant women & $437(89.2)$ \\
\hline b) eat a diet with higher iron content & $427(87.1)$ \\
\hline c) eat a diet with higher calcium content & $425(86.7)$ \\
\hline d) eat less fat & $353(72.0)$ \\
\hline 5) Smoking causes harm to the baby & $458(93.5)$ \\
\hline $\begin{array}{l}\text { 6) Folic acid supplementation and risk reduction } \\
\text { of congenital malformation }\end{array}$ & $344(70.2)$ \\
\hline \multicolumn{2}{|l|}{ 7) Risk of maternal anaemia for baby } \\
\hline a) low birth weight & $291(59.4)$ \\
\hline b) pale & $231(47.1)$ \\
\hline c) increased appetite & $53(10.8)$ \\
\hline d) increased blood pressure & $51(10.4)$ \\
\hline
\end{tabular}


Table 4: Factors associated with good pre-pregnancy care knowledge, using simple logistic regression analysis $(n=490)$

\begin{tabular}{|c|c|c|c|c|c|}
\hline \multirow[t]{2}{*}{ Variable } & \multicolumn{2}{|c|}{$\begin{array}{l}\text { Good pre-pregnancy care } \\
\text { knowledge }\end{array}$} & \multirow[t]{2}{*}{$b$} & \multirow[t]{2}{*}{ Crude OR $(95 \% \mathrm{Cl})$} & \multirow[t]{2}{*}{$p$-value } \\
\hline & $\begin{array}{c}\text { Yes } \\
\text { n (\%) }\end{array}$ & $\begin{array}{c}\text { No } \\
\mathrm{n}(\%)\end{array}$ & & & \\
\hline \multicolumn{6}{|l|}{ Age (year) } \\
\hline$<20$ and $>35$ & $43(18.1)$ & $51(20.2)$ & & 1 & \\
\hline 20 to 35 & $195(81.9)$ & $201(79.8)$ & 0.14 & $1.15(0.73,1.81)$ & 0.542 \\
\hline \multicolumn{6}{|l|}{ Ethnicity } \\
\hline Others & $2(0.8)$ & $2(0.8)$ & & 1 & \\
\hline Malay & 175 (73.5) & $172(68.3)$ & 0.02 & $1.02(0.14,7.31)$ & 0.986 \\
\hline Chinese & $27(11.4)$ & $38(15.0)$ & -0.34 & $0.71(0.09,5.36)$ & 0.740 \\
\hline Indian & $34(14.3)$ & $40(15.9)$ & -0.16 & $0.85(0.11,6.36)$ & 0.874 \\
\hline \multicolumn{6}{|l|}{ Education level } \\
\hline Secondary and below & $131(55.0)$ & $182(72.2)$ & & 1 & \\
\hline Tertiary & $107(45.0)$ & $70(27.8)$ & 0.75 & $2.12(1.46,3.09)$ & $<0.001$ \\
\hline \multicolumn{6}{|l|}{ Employment status } \\
\hline Unemployed & $90(37.8)$ & $127(50.4)$ & & 1 & \\
\hline Employed & $148(62.2)$ & $125(49.6)$ & 0.51 & $1.67(1.17,2.40)$ & 0.005 \\
\hline \multicolumn{6}{|l|}{ Monthly income (RM) } \\
\hline Less than 5000 & $159(66.8)$ & $194(77.6)$ & & 1 & \\
\hline 5000 to 10,000 & $70(29.4)$ & 49 (19.6) & 0.56 & $1.74(1.14,2.66)$ & 0.010 \\
\hline More than 10,000 & $9(3.8)$ & $7(2.8)$ & 0.45 & $1.57(0.57,4.31)$ & 0.382 \\
\hline \multicolumn{6}{|l|}{ Parity } \\
\hline Nulliparous & $66(27.7)$ & $85(33.7)$ & & 1 & \\
\hline Para 1 and above & $172(72.3)$ & $167(66.3)$ & 0.28 & $1.33(0.90,1.95)$ & 0.151 \\
\hline \multicolumn{6}{|l|}{ Gestational age at booking } \\
\hline$>12$ weeks & $63(26.5)$ & $63(25.0)$ & & 1 & \\
\hline 12 weeks and below & $175(73.5)$ & $189(75.0)$ & -0.08 & $0.93(0.62,1.39)$ & 0.710 \\
\hline \multicolumn{6}{|l|}{$\begin{array}{l}\text { Previous pregnancy } \\
\text { complications }\end{array}$} \\
\hline No & $95(39.9)$ & $122(48.4)$ & & 1 & \\
\hline Yes & $143(60.1)$ & $130(51.6)$ & 0.35 & $1.41(0.99,2.02)$ & 0.059 \\
\hline \multicolumn{6}{|l|}{ Planned pregnancy } \\
\hline No & $92(38.7)$ & $100(39.7)$ & & 1 & \\
\hline Yes & $146(61.3)$ & $152(60.3)$ & 0.04 & $1.04(0.73,1.50)$ & 0.816 \\
\hline \multicolumn{6}{|l|}{ Family planning practice } \\
\hline No & $162(68.1)$ & $206(81.7)$ & & 1 & \\
\hline Yes & 76 (31.9) & $46(18.3)$ & 0.74 & $2.10(1.38,3.20)$ & 0.001 \\
\hline \multicolumn{6}{|l|}{$\begin{array}{l}\text { Received advice on pre- } \\
\text { pregnancy care }\end{array}$} \\
\hline No & $150(63.0)$ & $191(75.8)$ & & 1 & \\
\hline Yes & $88(37.0)$ & $61(24.2)$ & 0.61 & $1.84(1.24,2.71)$ & 0.002 \\
\hline \multicolumn{6}{|c|}{$\begin{array}{l}\text { Referred for pre-pregnancy } \\
\text { care services }\end{array}$} \\
\hline No & 175 (73.5) & $212(84.1)$ & & 1 & \\
\hline Yes & $63(26.5)$ & $40(15.9)$ & 0.65 & $1.91(1.22,2.97)$ & 0.004 \\
\hline \multicolumn{6}{|l|}{$\begin{array}{l}\text { Attended pre-pregnancy } \\
\text { care services }\end{array}$} \\
\hline No & $161(67.6)$ & $212(84.1)$ & & 1 & \\
\hline Yes & $77(32.4)$ & $40(15.9)$ & 0.93 & $2.54(1.64,3.91)$ & $<0.001$ \\
\hline
\end{tabular}

Table 5: Factors associated with good pre-pregnancy care knowledge, using multiple logistic regression analysis $(n=490)$

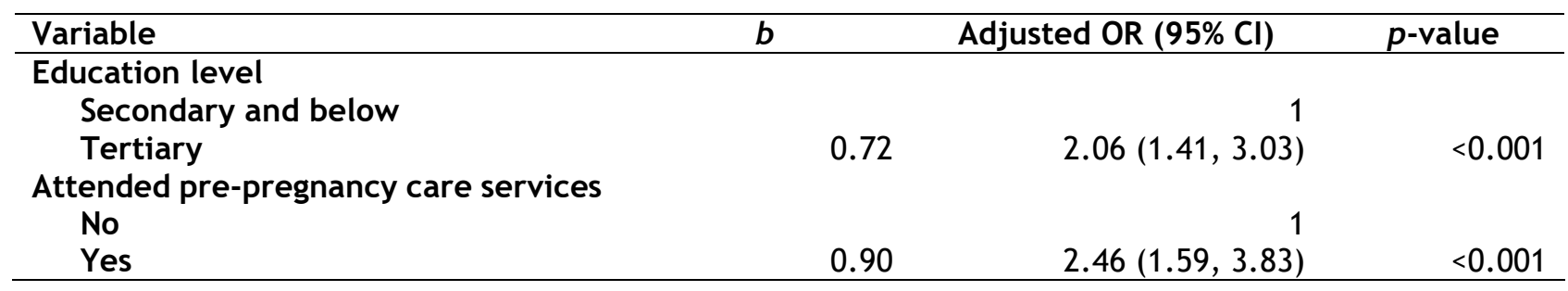




\section{DISCUSSION}

The age range of the respondents in this study was between 16 years old and 43 years old. There were 18 respondents $(3.7 \%)$ with the age of 19 years and below, and $76(15.5 \%)$ aged more than 35 years old. Various studies have shown the association between teenage pregnancy and advanced maternal age with adverse maternal and neonatal outcomes, including preterm delivery, eclampsia, postpartum haemorrhage and fetal distress ${ }^{13,18}$. Therefore, educating and empowering women are important to prevent too early, too late or too close pregnancies. One of the important strategies to achieve optimal timing and spacing of pregnancies is through prepregnancy care.

More than half of the respondents already had previous history of pregnancy complications. These women should prioritize the pre-pregnancy care and optimize their conditions before embarking into another pregnancy. Nonetheless, some high risk women were keen to get pregnant despite knowing the possible risks, and refuse for pre-pregnancy care or contraception use ${ }^{19}$. Focus group discussions with women who had chronic health conditions found that obese women generally did not perceive their increased risk for pregnancy-related complications, and obesity did not influence their pregnancy intentions ${ }^{20}$.

This study also demonstrated a low percentage of family planning use prior to the current pregnancy. Similarly, $80.8 \%$ of women who attended pre-pregnancy screening were found not practising contraception despite having risk factors ${ }^{6}$. This highlights an unmet need for family planning among women with pregnancy risk factors. It is closely linked to the issue of unplanned or unintended pregnancy. Almost $40.0 \%$ of women in this study had an unplanned pregnancy. Unplanned pregnancy is an important public health issue. A study in Sarawak showed that $55.0 \%$ of women never planned their pregnancies before, and unplanned pregnancy was expressed as one of the main barriers of prepregnancy care services ${ }^{19}$. Similarly, the major cause of lack of pre-pregnancy care expressed by women in Iran was unplanned pregnancy 21. However, some women did not view unintended pregnancy as a result of inadequate contraceptive use, but related it with fate or decision of $\mathrm{God}^{20}$. This perception and belief need to be tackled in order to improve family planning practice and pre-pregnancy care.

Using the same questionnaire, a study conducted among pregnant women in another state in Malaysia found an almost similar percentage $(51.9 \%)$ of the respondents who had good prepregnancy care knowledge ${ }^{16}$. Our study also showed that almost half of the women had poor knowledge. However, $61 \%$ of the population whom the questionnaire was first utilized were reported to have high score in knowledge on pre-pregnancy care $^{17}$. The possible explanation can be related to the difference in ethnic groups of the respondents. Their study only included Indian population residing in Selangor, whereas majority of our respondents were Malay. Nevertheless, the authors concluded that the level of knowledge among their study population was still low ${ }^{17}$. The inadequate knowledge of pre-pregnancy care was also demonstrated from other countries, including Nepal, where $51.0 \%$ of antenatal mothers had inadequate pre-pregnancy care knowledge, and majority of them had an average knowledge ${ }^{22-23}$.

Likewise, as found in our study, many women did not have adequate knowledge regarding optimal duration of birth spacing, risk of poor birth spacing, and the factors classified as high risk pregnancy ${ }^{16}$. Only $5 \%$ of reproductive age women in a study in Nepal were able to provide correct answer regarding the recommended minimum gap between two children ${ }^{23}$. Therefore, it is a need to highlight the risk of short and long birth spacing, in order to encourage women to have optimal birth spacing. One of the ways is to have adequate access to quality family planning services, which is also provided through pre-pregnancy care services.

It is important for all women, especially those in the reproductive age group, to have good prepregnancy care knowledge. Our study found that women with tertiary education and those who had attended pre-pregnancy care services were more likely to have good pre-pregnancy care knowledge. The association between educational level and pre-pregnancy care knowledge was demonstrated in other studies as well ${ }^{12,24}$. A community-based study among reproductive age women found that women with higher education level were more likely to have knowledge on prepregnancy care ${ }^{25}$. Therefore, health promotion on pre-pregnancy care should focus on all women, with more emphasis on those in lower educational group.

In addition, women who had the experience of attending pre-pregnancy care services before were found to be more knowledgeable on prepregnancy care. Knowledge of pre-pregnancy care may be acquired through experience or education ${ }^{16}$. Those who had been exposed and utilized the service might obtain the important and relevant information, thus their knowledge on pre-pregnancy care were better. The major source of information on pre-pregnancy care was from the health care providers, and they should be well-equipped with updated and correct information to be able to disseminate it to the general population?.

The findings from this study are beneficial for the improvement of the current health services. Knowing the level of knowledge, it is clear that pre-pregnancy care services should be made 
informed and accessible to all levels of the community. Disseminating information for the improvement of pre-pregnancy care knowledge should be achieved through various channels, including collaboration with the community and non-governmental organizations. Tackling the issue of unmet need for family planning is very important, and ensuring optimal birth spacing should be prioritized in every relevant program at the health clinics. In addition, pre-pregnancy care knowledge and family planning should be clearly highlighted during pre-marital courses. Apart from that, more research can be conducted to develop an effective and comprehensive module for pre-pregnancy care knowledge, with involvement of various stakeholders and suited for different level of population.

\section{Limitation}

This study was conducted among women who came for their antenatal booking in governmental health clinics. The findings might not represent those who went to government hospitals and private clinics or hospitals. Those women might have different level of knowledge related to prepregnancy care. Furthermore, the women in this study might already be exposed to some information related to pre-pregnancy during this booking visit. In addition, some knowledge items in the questionnaire could be assessed in more detail to get a clearer picture of their knowledge. Nevertheless, this study provides important insights into the general understanding of their knowledge and the associated factors. Further exploration of these findings can be conducted in future research.

\section{CONCLUSION}

The knowledge of women with high risk pregnancy about pre-pregnancy care was still unsatisfactory. There were high proportion of them who were not aware of the recommended duration of birth spacing, the risk of poorly spaced birth, and the pregnancy conditions classified as high risk. The factors significantly associated with good prepregnancy care knowledge were women who received tertiary education and attended prepregnancy care services before.

\section{ACKNOWLEDGEMENT}

We gratefully acknowledge Universiti Sains Malaysia for the research grant (Universiti Sains Malaysia Research University Grant, 1001.PPSP.812212) and Ministry of Health Malaysia for allowing us to conduct the study. We also like to thank all the women who had participated, and individuals who were directly and indirectly involved in this study.

\section{Conflict of interest}

The authors declare no potential conflict of interest.

\section{REFERENCES}

1. World Health Organization 2016. WHO recommendations on antenatal care for a positive pregnancy experience.

2. World Health Organization 2013. Preconception care: Maximizing the gains for maternal and child health.

3. Jourabchi Z, Sharif S, Lye MS, et al. Association between preconception care and birth outcomes. Am J Health Promot 2019; 33(3):363-71.

4. Ministry of Health Malaysia 2015. Prepregnancy care (PPC). Retrieved from www.myhealth.gov.my (accessed January 2019).

5. Ministry of Health Malaysia 2013. Perinatal Care Manual $3^{\text {rd }}$ edition.

6. Nik Mazlina M, Ruziaton $H$, Nuraini DB, et al. Risk factors for women attending prepregnancy screening in selected clinics in Selangor. Malays Fam Physician 2014; 9(3):206.

7. Yeoh PL, Hornetz K, Dahlui M. Antenatal care utilisation and content between low-risk and high-risk pregnant women. PLoS One 2016; 11(3):e0152167.

8. Abu Talib R, Idris IB, Sutan R, et al. Patterns of pre-pregnancy care usage among reproductive age women in Kedah, Malaysia. Iran J Public Health 2018; 47(11):1694-1702.

9. Demisse TL, Aliyu SA, Kitila SB, et al. Utilization of preconception care and associated factors among reproductive age group women in Debre Birhan town, North Shewa, Ethiopia. Reproductive Health 2019; 16:96.

10. Barret G, Shawe J, Howden B, et al. Why do women invest in pre-pregnancy health and care? A qualitative investigation with women attending maternity services. BMC Pregnancy Childbirth 2015; 15:236.

11. Steel A, Lucke J, Adams J. The prevalence and nature of the use of preconception services by women with chronic health conditions: an integrative review. BMC Womens Health 2015; 15:14.

12. Olewekre AE, Komolafe A, Owofadeju C. Awareness, knowledge and uptake of preconception care among women in Ife Central Local Government Area of Osun State, 
Nigeria. Journal of Community Medicine and Primary Health Care 2015; 27(2):83-92.

13. Kassa A, Human SP, Gemeda H. Knowledge of preconception care among healthcare providers working in public health institutions in Hawassa, Ethiopia. PLoS One 2018; 13(10): e0204415.

14. Abu Talib R, Idris IB, Sutan R, et al. Exploring the determinant of pre-pregnancy care services usage among reproductive ages women in Kedah, Malaysia. International Journal of Public Health Research 2016; 6(2):719-726.

15. Rahim NA, Rahman MM. Barriers, strength and weakness of pre-pregnancy clinic services in Sarawak: a qualitative analysis from provider perspectives. Malaysian Journal of Public Health Medicine 2018; 18(1):149-157.

16. Kasim R, Draman N, Kadir AA, et al. Knowledge, attitudes and practice of preconception care among women attending appointments at a rural clinic in Kelantan. Education in Medicine Journal 2016; 8(4):5768.

17. Rosnah S, Wan Aishah WN. Pre-pregnancy care among the Indian ethnic group in Semenyih, Selangor. Journal of Community Health 2010; 16(2):29-35.

18. Cavazos-Rehg PA, Krauss MJ, Spitznagel EL, et al. Maternal age and risk of labor and delivery complications. Matern Child Health J 2015; 19(6): 1202-1211.

19. Rahman M, Rahim NA, Arif MT. Barrier, weakness and utilization of pre-pregnancy clinic services. Arch Public Health 2017; 75:67.

20. Chuang $\mathrm{CH}$, Velott DL, Weisman CS. Exploring knowledge and attitudes related to pregnancy and preconception health in women with chronic medical conditions. Matern Child Health J 2010; 14(5):713-719.

21. Shadab P, Nekuei N, Yadegarfar G. The prevalence of preconception care, its relation with recipients' individuality, fertility, and the causes of lack of checkup in women who gave birth in Isfahan hospitals in 2016. J Educ Health Promot 2017; 6:88.

22. Nepali G, Sapkota SD. Knowledge and practice regarding preconception care among antenatal mothers. International Journal of Perceptions in Public Health 2017; 1(4):224227.

23. Gautam P, Dhakal R. Knowledge on preconception care among reproductive age women. Saudi Journal of Medical and Pharmaceutical Sciences 2016; 2(1):1-6.

24. Giri K. Knowledge on preconception care among reproductive aged women in Kaski district, Nepal. Janapriya Journal of Interdisciplinary Research 2018; V11:46-56.

25. Ayalew $Y$, Mulat A, Dile $M$, et al. Women's knowledge and associated factors in preconception care in adet, west gojjam, northwest Ethiopia: a community based cross sectional study. Reproductive Health 2017; 14:15. 